\title{
Kommentar zu Randall Collins' ,German-Bashing and the Theory of Democratic Modernization“ (ZfS 24: 3-21)
}

\section{Wolfgang Knöbl}

FU Berlin, John F. Kennedy-Institut, Lansstr. 5-9, D-14195 Berlin

Mißverständnisse beseitigen, Vorurteile abbauen, vernünftige Kommunikation herstellen - wer wollte solche hehren Ziele nicht umstandslos positiv beurteilen und auch tatkräftig unterstützen! Unternimmt es deshalb ein international renommierter amerikanischer Soziologe wie Randall Collins, das in anglo-amerikanischen Massenmedien verbreitete düstere Bild „der“ Deutschen (Nazis!) aufzuhellen und dem verbalen „Draufhauen" auf die deutsche Kultur, die angeblich in ihrem Kernbestand immer schon durchgängig antidemokratische und vormoderne Merkmale trug und noch trägt, entgegenzutreten, so wird ein solcher Versuch sicherlich ganz spontan auf die überwiegende Zustimmung und Unterstiutzung gerade des deutschen Publikums stoßen. Der ursprünglich wohl für amerikanische Leser geschriebene Text enthält allerdings einige sachlich höchst problematische Thesen, die es zu korrigieren gilt, wenn das von Collins erstrebte Ziel der „Völkerverständigung“ erreicht werden soll.

Collins' Argumente zielen kritisch auf demokratische Entwicklungsvorstellungen wie sie überwiegend (aber natürlich nicht nur) der evolutionistischen Modernisierungstheorie zugrundegelegen haben und zugrundeliegen. Die Entstehung der nationalsozialistischen Gewaltherrschaft in Deutschland wurde in dieser Theorietradition zum Anlaß genommen, nach Demokratiedefiziten in der deutschen Gesellschaft lange vor 1933 zu suchen, die dann auch gefunden wurden und die sich zum Urteil der Rückständigkeit dieser deutschen Gesellschaft und der Demokratiefeindschaft von Teilen ihres kulturellen Erbes verdichteten. Collins hält dies für eine falsche Diagnose, die nur aus einer Art teleologischer Geschichtsbetrachtung zu erklären ist, der allzu viele Strukturen und Ereignisse der deutschen Geschichte des 19. Jahrhunderts zu Ursachen des späteren Faschismus geraten.

Für ihn war es vielmehr ein kontingenter Faktor, der Erste Weltkrieg und sein Ausgang (S. 19), der die entscheidende Weichenstellung für die spätere nationalsozialistische Machtergreifung darstellte: Der verlorene Krieg, die von den Rechten vehe- ment betriebene Delegitimierung der politischen Eliten (,Dolchstoßlegende“), die ökonomischen Probleme der Weimarer Republik usw. usw. hätten erst den Aufstieg der NSDAP möglich gemacht. Den deutschen politischen Verhältnissen und Strukturen vor der instabilen Phase des Ersten Weltkrieges und der Weimarer Republik wird von Collins nicht nur ein determinierender Einfluß, sondern überhaupt jegliche Relevanz für spätere Entwicklungen abgesprochen. Die Suche nach belastenden kulturellen Traditionen und sozialstrukturellen Verhältnissen, welche die Krisenhaftigkeit der deutschen Politik im ersten Drittel des 20. Jahrhunderts und die daraufhin erfolgte Errichtung der nationalsozialistischen Gewaltherrschaft ja auch möglich gemacht haben könnten, wird von Collins für fruchtlos gehalten.

Damit dies keine unbegründete und willkürliche Position bleibt, muß Collins zeigen können, daß Deutschland vor 1918 (oder 1933) im Hinblick auf seine Modernität mindestens ebenso entwickelt war wie vergleichbare westliche Länder. Nur dann läßt sich nämlich seine These halten, daß es ausnahmslos kontingente Bedingungen unmittelbar vor 1933 waren (der verlorene Weltkrieg und die soziale und ökonomische Krise der Weimarer Republik), welche die Herrschaft der Nazis möglich werden ließen.

Collins tritt seine Beweisführung mit Hilfe eines analytischen Rasters an, das den umfassenden Modernisierungsprozeß in vier Teilprozesse (Bürokratisierung, Säkularisierung, kapitalistische Industrialisierung und Demokratisierung) zerlegt. Tatsächlich gelingt es ihm in bezug auf die ersten drei Dimensionen des Modernisierungsprozesses mühelos, die Modernität der deutschen Gesellschaft des 19. Jahrhunderts nachzuweisen, wobei die hier von Collins vorgebrachten Argumente in der historisch-soziologischen Fachliteratur allerdings auch wenig umstritten sind. Es ist kaum kontrovers, daß die zu Beginn des 19. Jahrhunderts besonders in Bayern und Preußen begonnenen und vorangetriebenen Verwaltungsrationalisierungen in einer gewissen Weise "modern" waren, daß die preußische Bürokratie hinsichtlich ihrer Funk- 
tionsfähigkeit anderen Ländern als Vorbild diente und damit alles andere als rückständig war. Das gleiche läßt sich auch im Hinblick auf die kapitalistische Industrialisierung festhalten: Es überrascht nicht, wenn Collins feststellt, daß Deutschland den industriellen Vorreiter England gegen Ende des 19. Jahrhunderts eingeholt hatte und zu einer der führenden Industrienationen geworden war. Und auch Collins' Ausführungen zur Säkularisierung sind in ihren inhaltlichen Aspekten wenig umstritten, wenn er beispielsweise darauf hinweist, daß gerade in Deutschland Schulen und Universitäten vergleichsweise schnell von religiöser Bevormundung befreit wurden und in ihrer so gewonnenen "Modernität" wiederum zum Vorbild für andere westliche Länder (und nicht nur für diese) werden konnten.

All diesen eher wenig kontroversen Analysen folgt dann aber das weitere - das entscheidende - Argument Collins', nämlich daß Deutschland auch in der Demokratisierungsdimension des Modernisierungsprozesses keine Defizite gegenüber den anderen westlichen Nationen aufzuweisen hatte. Dies ist nun alles andere als eine unumstrittene Position, richtet sie sich doch ganz explizit gegen Vertreter der These des deutschen Sonderweges, die immer wieder fast formelhaft auf das Auseinanderlaufen der wirtschaftlichen und politischen Entwicklung in Deutschland aufmerksam gemacht hatten: Der rapiden ökonomischen und industriellen Entwicklung sei keine entsprechende politische Modernisierung, d.h. keine adäquate Demokratisierung und Parlamentarisierung des politischen Lebens gefolgt.

Collins greift diese These (allerdings ohne zugehörige Autoren zu nennen und ohne die Entwicklung der Sonderwegsdebatte zu berücksichtigen) fundamental an, indem er zu zeigen versucht, daß Deutschland im 19. Jahrhundert mindestens ebenso stark demokratisiert war wie etwa Frankreich, England oder die USA - eine scharfe Attacke, die er unbedingt deshalb vortragen muB, damit er seine schon genannte These von der Kontingenz des Aufstiegs des Nationalsozialismus behaupten kann. Als Demokratisierungsindikatoren verwendet Collins hierbei ausschließlich das Wahlrecht (,the proportion of the populace which participates in the political franchise", S. 15) und die Existenz von kollegial organisierten Machtorganen (,the extent of collegially shared power [through parliaments, councils, and other such structures]", S. 15). Wenn man wie Collins genau diese und nur diese Maßstäbe anlegt, erhält man tatsächlich ein Bild, das Deutschland keineswegs als „politisch rückständig“ gegenüber anderen Nationen zeigt. Mit der Institution des Reichstages und dem Institut des allgemeinen Männerwahlrechts war bereits im Bismarckreich ein im europäischen Maßstab durchaus üblicher Verfassungsaufbau gewährleistet. Es fragt sich allerdings, ob allein mit diesen Indikatoren die Lage der Demokratie im zeitgenössischen Deutschland adäquat gekennzeichnet werden kann. Zum einen werden damit nämlich Fragen der institutionellen Machtverteilung weitgehend abgeblockt - Fragen nach der tatsächlichen Macht von Parlamenten, der Unabhängigkeit und Eigenständigkeit von Verfassungsinstitutionen, der Versäulung der politischen Parteien aufgrund ethnischer, religiöser oder schichtenmäßiger Bindungen usw. Das kaiserliche Deutschland, in dem der Reichstag auf die Regierungsbildung wenig Einfluß hatte, dessen politisches Leben durch eine äußerst scharfe Frontstellung gegenüber Sozialisten und Katholiken gekennzeichnet war, schneidet bei der Hinzuziehung dieser Merkmale im Vergleich zur zeitgenössischen anglo-amerikanischen Welt bekanntlich nicht allzu günstig ab - aber das sind Merkmale, die durch Collins' analytisches Raster erst gar nicht erfaßt werden.

Zum anderen werden durch die ausschließliche Verwendung der von Collins vorgeschlagenen Demokratisierungsindikatoren alle normativen Implikationen des Demokratiebegriffs, so wie er in der Moderne entwickelt wurde, eskamotiert, was Collins allerdings auch durch seine Rede vom nichtmodernen Charakter der Demokratie bewußt in Kauf zu nehmen scheint (,It may well be that democracy is not inherently very modern, indeed that it goes against the grain of other features of modern social structure.“, S. 6). In der Tat ist es so, daß sich allein durch die Konzentration auf den Aspekt der Mehrheitsentscheidung (und/oder des Wahlrechts) zu jeder historischen Epoche irgendwelche Vorformen „demokratischer" Strukturen auffinden lassen (Collins sieht sowohl in Jägerund Sammlergesellschaften als auch im Wahlkönigtum [vor-]demokratische Strukturen verankert, sofern nur kollektive Entscheidungsgremien vorhanden waren, ibid). Verloren geht dabei unter anderem aber die Einsicht, daß sogar zwischen dem antiken griechischen Demokratieverständnis und dem modernen westlichen durchaus fundamentale Unterschiede bestehen ${ }^{1}$ - Unterschiede die sich mit den Begriffen der politischen Freiheit, der rechtlichen Gleichheit und der persönlichen Auto-

\footnotetext{
1 Dies wurde zuletzt energisch von Hauke Brunkhorst (1994) herausgearbeitet.
} 
nomie aller Menschen bezeichnen lassen. Diese demokratischen Grundwerte wurden erst in der Moderne - und nicht schon vorher! - voll entwickelt und in den sogenannten „bürgerlichen“ oder „demokratischen“ Revolutionen des 18. Jahrhunderts institutionell (wie unvollkommen auch immer) festgeschrieben (vgl. Preuß 1990: 15 ff.). Diese Werte waren nicht bloße „Zutaten" zur Mehrheitsregel oder zum Wahlrecht, sondern mindestens ebenso wichtig wie diese selbst, prägt doch noch heute der Kampf um ihre weitgehende rechtliche Durchsetzung und gesellschaftliche Verwirklichung fundamental den politischen Proze $B$ und die politische Entwicklung, obwohl die von Collins benannten beiden demokratischen Grundprinzipien im Westen bereits lange verwirklicht worden sind. Collins verbaut sich mit seinem verengten Demokratieverständnis nicht nur den Einblick in die Dynamik demokratischer Prozesse. Er versperrt sich auch einen realistischen Einblick in die Verwirklichung demokratischer Werte, so daß seine vergleichenden Aussagen über die Lage der Demokratie in Deutschland oder anderswo zwar wichtige, aber sicher nicht alle wichtigen Aspekte in Betracht ziehen. Seine These von der „normalen" demokratischen Entwicklung in Deutschland kann deshalb nicht ernsthaft überzeugen.

Ähnlich kritische Anmerkungen lassen sich auch zu seiner Auffassung von der Bedeutung von Revolutionen für den Demokratisierungsprozeß machen. Natürlich ist die Beziehung zwischen Revolution und demokratischer Entwicklung keine einfache, münden viele Revolutionen in Diktaturen usw. Selbst wenn beispielsweise die Französische Revolution in die Diktatur Robespierres und $\mathrm{Na}$ poleons führte, so prägte sie aber doch sowohl die Verfassungsentwicklung als auch das politische Denken und nicht zuletzt die politische Kultur der jeweiligen Länder. Es ist und war deshalb kein völlig abseitiger Gedanke, wenn Sonderwegstheoretiker auf die Bedeutung dieser Revolutionen des 18 . Jahrhunderts für die Verfassungsentwicklung in den entsprechenden Ländern und auf das Fehlen von ,echten“ Revolutionen in Deutschland hinwiesen, wie stark man diesen Faktor dann schlieBlich auch immer gewichten mag: Die deutschen Revolutionen von 1848 und 1918/1919 - und schon gar nicht die von Collins angeführten ,revolutionären" preußischen Reformen von 1807-14 (S. 15) - erreichten niemals die Bedeutung für das politische Selbstverständnis der Bürger, wie es die $\mathrm{Re}$ volutionen etwa in den USA oder in Frankreich taten, was nicht ohne Folgen für die politische Konfliktaustragung und damit die weitere politische
Entwicklung blieb. Die Beschwörungen der Ideale der Revolution stellten nicht selten Phrasendrescherei zur Verdeckung handfester materieller Interessen dar. Aber sie waren dies nicht ausschließlich, und vor allem: die Phrasen hatten Konsequenzen, vor allem dann, wenn es um die Ausgestaltung verfassungsrechtlicher Ordnungen ging oder wenn Unterschichten plötzlich begannen, die revolutionären Ideale auch wirklich einzufordern.

Legt man einen umfassenderen Demokratiebegriff als den von Collins verwendeten zugrunde, so dürfte es durchaus möglich sein, gewisse politische Traditionsbestände in Deutschland zu entdecken, die das 19. Jahrhundert überdauert und auch die Errichtung der späteren Diktatur möglich gemacht haben, wenngleich natürlich von Determination keine Rede sein kann: die geringe Ausstrahlungskraft des Bürgertums, die Übermacht der Bürokratie oder eine früh sich abzeichnende spezifisch anti-liberale Form des Nationalismus mögen hier als Stichworte genügen, um anzuzeigen, daß es durchaus langwirkende Traditionen gab, welche später dann die Überlebensfähigkeit der Demokratie Weimars nicht gerade stärkten - Traditionen, die es in dieser Form und in dieser spezifischen Mischung in den westlichen Ländern nicht gab (vgl. vorbildhaft hierzu Kocka 1989). Collins' provokante These von der radikalen Kontingenz des Aufstiegs des Nationalsozialismus steht angesichts der hier nur angedeuteten Besonderheiten der politischen Kultur und Verfassungsentwicklung Deutschlands auf schwachen Füßen.

Es gab natürlich keinen uranfänglichen antidemokratischen Keim der deutschen Kultur, aus dem sich zwangsläufig der Nationalsozialismus entwickelt hätte. Darin ict Collins sicherlich zuzustimmen. Andererseits macht es wenig Sinn, dieser Kontinuitätsthese eine ebenso radikale der Diskontinuität und Kontingenz entgegenzusetzen. Die deutsche Geschichte war von Kontinuitäten und Diskontinuitäten (Nipperdey 1991) geprägt. Diese eher triviale Einsicht bleibt zu berücksichtigen und ist argumentativ auch einzulösen, wenn man mit Aussicht auf Erfolg aufklären und damit dann auch ein Ende des "German-Bashing" herbeiführen will.

\section{Literatur}

Brunkhorst, H., 1994: Demokratie und Differenz. Vom Klassischen zum Modernen Begriff des Politischen. Frankfurt/M.: Fischer. 
Kocka, J., 1989: Deutsche Geschichte vor Hitler. Zur Diskussion über den "deutschen Sonderweg“. S. 101-113. in Ders., Geschichte und Aufklärung. Göttingen: Vandenhoeck \& Ruprecht

Nipperdey, Th. 1991: 1933 und die Kontinuität der deutschen Geschichte. S. 225-248. in Ders., Nachdenken über die deutsche Geschichte. Essays. München: DTV.
PreuB, U.K. 1990: Die Verfassung als „Gegenstand allen Sehnens". S. 11-21. in Ders., Revolution, Fortschritt und Verfassung. Zu einem neuen Verfassungsverständnis. Berlin: Wagenbach. 